\title{
The Journal of the \\ American Medical Association
}

Published under the Ausplces of the Board of Trustees.

\section{Original Articles \\ COCCIDIOIDAL GRANULOMA.}

REYIEW OF TILE EIGHTEEN CASES AND REPORTS OF CASES FIFTEEN AND SIXTEEN.

PHILIP KING BROWN, M.D.

SAN FRANCISCO.

The recognition, within a few years, of a comparatively large number of extremely fatal cases of a hitherto unknown disease, seems of sufficient interest to warrant me in presenting the last of these cases, together with a history of the findings in the other cases, the localities from which they came and a statement of certain experimental data in regard to the possible modes of infection.

A review by Ricketts ${ }^{1}$ on oidiomycosis gives an interesting statement of the pathogenicity of the moulds that belong to this class and a discussion of their relation to each other. He dwells at great length on the cases of blastomycetic dermatitis, occurring in the middle West, reviews fourteen old cases of this disorder and reports several more which he has collected. He also reviews the cases of coccidioidal granuloma and considers that there is a close relation between it and blastomycetic dermatitis and also Busse's saccharomycosis hominis.

Ophuls ${ }^{2}$ reviews the published cases of coccidioidal granuloma, and in 1905, at the annual session of the American Medical Association, gave in a supplementary paper the cases that have occurred since. He pointed out very plainly certain essential differences between this disease and blastomycetic dermatitis and reviewed the morphologic and histologic studies of the condition so thoroughly, that it seems unnecessary to go into them here. It is sufficient to state that in coccidioidal granuloma there are certain characteristics which differ decidedly from blastomycetic dermatitis. The differences in the behavior of the two organisms on culture media are very striking; even in the organisms of blastomycetic dermatitis, which most closely resemble those of coccidioidal granuloma, there are essential differences. It must be apparent that no one mould is responsible for the various lesions which Ricketts groups under the name of blastomycetic dermatitis, and he makes clear in his tabulation of the morphologic characteristics that some resemble the organism of coccidioidal granuloma more than others do. On agar the growths of the coccidioidal granuloma mould are all sharply defined even when grown on dry media where the mycelia are especially profuse. This is not true of any of the growths from blastomycetic dermatitis. The mould from coccidioidal granuloma is never found in the budding state in tissue. although in pus withdrawn from abscess cavities, beautiful examples of its growth by budding are shown. Cutaneous lesions are often entirely secondary

1. Journal of Medical Research, vol. vi. No. 3 .

2. Tournal of Experimental Medicine. vol. vi. in coccidioidal granuloma and may even be wanting altogether. Generalized infections are the rule and the only patient known positively to be still alive, is a man with lesions in the ankle, whose foot was promptly amputated. He has been reëxamined within a few weeks and at the time there were no signs of any further involvement of the body. Two of the patients were discharged from the hospital benefited, but nothing has been heard of them since and it seems probable that they could have been traced were they still alive. Only one of the cases of blastomycetic dermatitis became subsequently a generalized infection and the patient died from the disease. There is good evidence that cases of blastomycetic dermatitis have been held in abeyance or cured by potassium iodid. This has been very thoroughly tried in coccidioidal granuloma in a number of cases ( I can speak most positively for the one I am reporting), and there was not the slightest benefit from it.

Lesions of coccidioidal granuloma are almost invariably progressive, with marked tendency to dissemination by lymph and blood currents. In very rare instances, healed lesions have been observed and in a few of the cases the course of the disease was extremely low. It is probable that the skin lesions were primary in some of the cases, particularly as in those cases where skin manifestations were noted before other symptoms, they occurred always on exposed parts.

Attempts were made to transmit the disease to guinea-pigs through original cultures from Case 15, in the following manner: Cultures known to have reached the spore-forming stage were transferred to the nostrils of pigs who were kept in glass cases. Another set of pigs were fed on similar cultures. A male pig was inoculated intraperitoneally and because of the characteristic genital lesions in pigs so inoculated, as pointed out by Ophuls, the experiment of transmission was tried by placing him in a cage with female pigs.

The secondary skin lesions are undoubtedly due to emboli in the blood current and of course occur anywhere. So far as I know no blood cultures have been attempted and it is hoped that this may be done in the cases that are likely to follow. The diagnosis of the disease is so easy after one has once known of its existence that ample opportunity for studies of the infectious nature of the blood must present itself. I regret exceedingly not having tried it in Case 15.

CAsE 15.-S. K., aged 25, Japanese laborer, consulted me Feb. 22, 1906 .

Past History.-His past history is negative to time of leaving Japan in 1903. He lived in Honolulu two years, came to California in June, 1905, and entered the employ of the Southern Pacific Company at Bakersfield. He worked in an extra gang along the line from Bakersfield to Tulare and came to the hospital from Tulare, where he had been working for a month. While in Bakersfield, he had what was regarded as beriberi for a month; the attack was not severe enough to keep him from work. He recovered from this and felt well up to Nor. 9, 1905. Previous to this he had good health. He 
never had syphilis or gonorrhea; he does not remember having had a break in the skin at any time, certainly he has had no skin sores.

Present Illness.-On Nov. 9, 1905, he had a vomiting spell and felt feverish. He thought he had caught cold, on account of a troublesome cough. He came to the Southern Pacific Hospital on Nov. 13, 1905, complaining of pain in his chest, a cough, constipation, slight headache, with pain in right ankle and left sternoclavicular articulation. The pain was not severe or constant and occurred only on moving the joints. Temperature on admission was 100.8 .

Examination.-Nutrition was good. Tongue was slightly coated. Heart sounds were normal. There was normal resonance over the chest. Abdomen was normal. No cutaneous lesions could be seen anywhere. There was no enlargement of glands. There was an old hydrocele on the left side. None of his joints was swollen or sensitive to touch and there was no impairment in function in any of his joints. Nerve tracts were not tender and there was no edema.

Course of Disease.-During the first week in the hospital the pain began to be more severe and more constant in the right

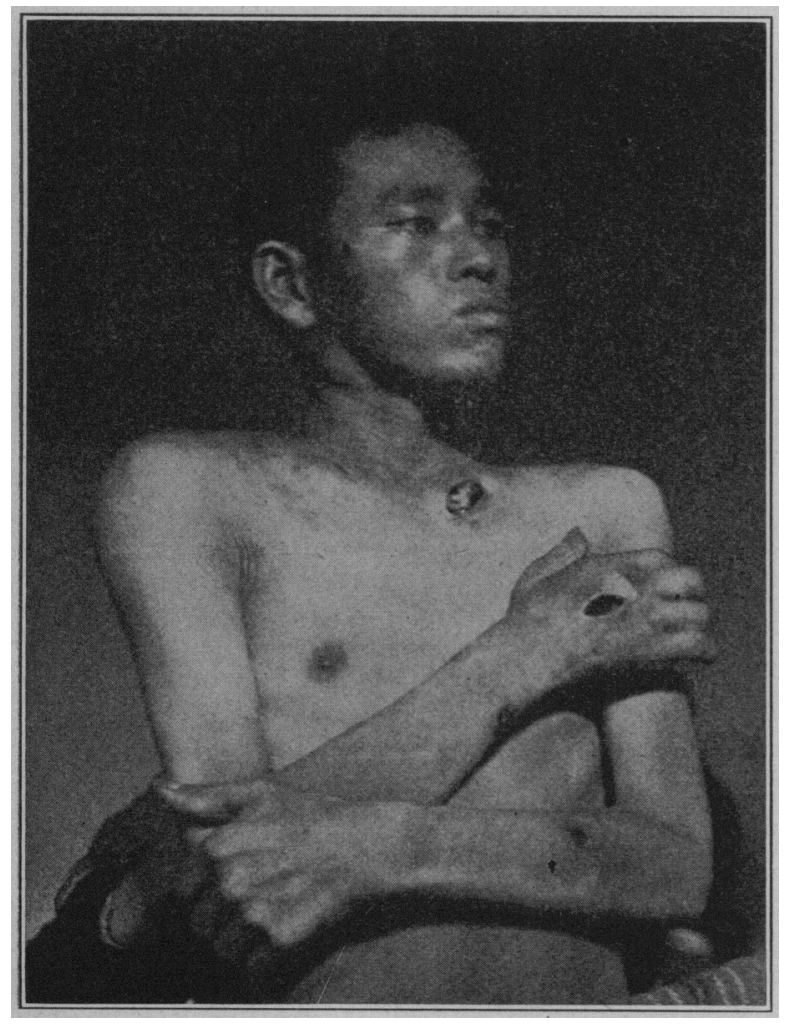

Fig. 1.-Case 15.

ankle and left sternoclavicular articulation. These joints were not red or tender. Other joints became painful on movement, the right wrist and left clavicularscapular articulations being attacked successively. Four weeks later a fluctuating swelling appeared in the region of the right internal malleolus. This was followed within a period of twelve or fourteen days, by the appearance of similar fluctuating swellings in the following places successively: Dorsum of right hand; right supraclavicular fossa; left supraspinous fossa; left sternoclavicular articulation. At this time the right inguinal, the epitrochlear glands on both sides and the right cervical glands became enlarged. The swellings developed with great rapidity for two or three days, but after that did not apparently increase in size or change in appearance or consistency. There was no redness, tenderness or edema of the skin covering them. There was no pain at any time at the swelling over the right supraclavicular fossa. At the right sternoclavicular articulation crepitation could be elicited. The swelling over the right internal malleolus was incised and a seropurulent fluid evacuated. Necrotic bone was found to exist. (In order to get a specimen free from contamination the swelling on the dorsum of the hand was aspirated and smears made. Examination was negative.) A few days later the swellings over the right supraclavicular fossa, left sternoclavicular articulation and left supraspinous fossa were incised and curetted. The left sternoclavicular articulation was found to be necrotic and also the the inner end of the clavicle. The spine of the scapula was found to be necrotic. The cavity in the right supraclavicular fossa did not communicate with the bone at any time, but extended between the layers of fascia toward the right pleural cavity. The wounds were packed with iodoform gauze, saturated with iodoform emulsion. Specimens of the pus and necrotic tissue were submitted to the laboratory, and pure cultures of the mould of coccidioidal granuloma obtained. A clinical diagnosis was made by several doctors before this.

Patient lost steadily in weight and there was considerable anorexia. Bowels were regular at this time (eighth week of disease). Cutaneous lesions appeared (having no connection with bone or joints) over the right malar bone, on the chin about an inch on left side of the symphysis, posterior surface of left forearm, on posterior surface of right forearm and on right thigh. They began as papillæ, which became converteil into flat papillary tumors, circular in shape, covered with crusts. On removal of the crust, pus escaped and a shallow ulcer was seen with an uneven floor, which bled easily on being touched. These ulcers continued to increase in size and in the case of the one on the chin, in depth, until it reached nearly to the bone. The patient's temperature was variable, at times normal, then, again 99 in the morning and 100 or 101 in the evening. During the last two weeks it was higher, sometimes 100 in the morning and 102 or 103 in the evening. During the last 48 hours the abdomen became distended and the temperature was 104 in the evening. No special tenderness.

Urinalysis: December 30: 1020; acid; trace albumin; few hyaline and fine granular casts.

Blood Examination: Jan. 1, 1906. Reds, 2,500,000; whites, 13,000; 79 per cent. polymorphonuclears; 17 per cent. small; 2 per cent. large; 2 per cent. eosinophiles; hemoglobin, 50.

Treatment.-Patient was given potassium iodid in increas. ing doses three times a day and tonics. No benefit followed medication. The man died Jan. 23, 1906.

Autopsy Report by Dr. Mary Halton.-Body of a fair-sized Japanese male. Condition of nutrition very poor. Small superficial ulceration about the size of a ten-cent piece over anterior half of right zygoma. There is conspicuous absence of redness or infiltration around the ulceration and this is true of every lesion found on this patient. The floor of the ulcer is of a dirty gravish color and is moderately moist. The ulceration is very shallow and lacks any punched out appearance. Practically no red granulation tissue is to be seen.

On left side of chin is a small, incised, rather superficial abscess. This contains a small quantity of creamy yellow pus. There is no redness or thickening whatever about the abscess. Abscess wall is of a yellowish white color and rather thin. No connection was found between this abscess and the bone. Many cervical glands both anterior and posterior on right side enlarged, movable, rather soft. Largest about the size of a hazelnut. Incised they appear to be very largely broken down into a thick yellowish pus.

In right supraclavicular fossa is an incision about $6.5 \times 10 \mathrm{~cm}$. long. This leads right into an irregular labyrinthine abscess cavity burrowing between the layers of cervical fascia in many directions, more especially downward. There is a large quantity of thick, yellow pus in this abscess cavity. Upper and inner surface of inner third of clavicle is eroded.

There is an incision about $5 \mathrm{~cm}$. long over left sternoclavicuiar articulation running out along the inner third of left clavicle. Sternoclavicular joint is disintegrated and the clavicle is necrotic for a distance of about half an inch. Sternum is also eroded. Cavity contains much thick pus. Incised abscess in outer half of left supraspinous fossa. The abscess extends outward toward acromion process, and outer third of spine of scapula shows superficial necrosis. This abscess also shows much thick creamy pus.

Dorsal surface of right hand shows large incised abscess cav. ity. There is some edema extending over metacarpal region of 
this hand. 'Third and fourth metacarpal bones show extensive superficial erosion. Same creamy pus found in this cavity. On extensor surface, upper third left forearm is a small, shallow uiceration about $2 \mathrm{~cm}$. in diameter. The description of ulceration over zygoma will exactly correspond to the description of this ulcer. There is a similar, small ulceration on lower third extensor surface right forearm. Epitrochlear glands palpable, largest on right side. On right thigh, outer side, upper third, is a small ulceration about $2.5 \mathrm{~cm}$. in diameter. The floor of this ulcer, unlike the others, shows a few red granulations, otherwise it is practically the same.

Right ankle is much swollen. There is a large incision over internal malleolus. A sinus leads into ankle joint which contains about 10 c.c. of thick creamy pus. The internal malleolus is about half gone, what remains shows an eroded surface. The astragalus and calcaneum show a roughening at edges of articulations. None of the bone lesions were of the soft spongy type. The bone left in all cases was very hard and showed an eroded surface.

In apex of right pleural cavity is a small abscess about the size of a dime, pointing into the pleural cavity and only covered by a thin layer of parietal pleura. With slight trauma this abscess was made to rupture into the pleural cavity, and the abscess was then found to be continuous with the large burrowing abscess of the neck on that side. A few adhesions were seen between the apex of right lung and sternoclavicular articulations. No fluid in right pleural cavity. Mediastinal glands enlarged, largest about the size of almond. Left lung shows adhesions light and friable anteriorly and posteriorly; adhesions much thicker in apex near abscess above. About 10 c.e. of blood-tinged fluid in left pleural cavity. Right primary bron chus filled with pus-like fluid. No particular infection of bronchus. Weight of lung, 18 ounces ; size, $23 \times 16 \times 5.5 \mathrm{~cm}$. Lung somewhat edematous. Left lung, slight hypostasis and edema, otherwise apparently normal. Weight, 17 ounces; size, $23.5 \times 14 \times 5.5 \mathrm{~cm}$.

Abscess in one of the peribronchial glands.

Heart.-About 10 c.c. blood-stained fluid in perieardium. Weight, 91/ ounces. Valves normal. Muscles pale, otherwise normal.

Abdomen.-Lower border transverse colon at umbilicus.

Liver.-One inch below costal margin in mammary line. Upper border fifth rib.

Diaphragm.-Right side, fifth rib. Left side, fifth rib.(?) Intestines.-Much distended. About 25 c.c. of seropurulent fluid in right iliac fossa. About 5 c.c. of thicker pus in rectal region. Appendix normal. Many heavy flakes of fibrinous exudate scattered over intestines, more marked in right iliac region. Conspicuous lack of injection of peritoneal surfaces, also a conspicuous lack of adhesions between the viscera.

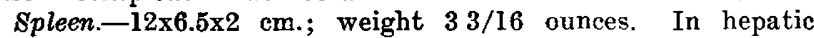
fossa is about 50 c.c. of pus, held as an abscess by adhesions.

Liver.-Surface covered by heavy fibrinous deposits; weight, 3 pounds $4 \frac{1}{2}$ ounces; size, $29 \times 14 \times 7.75 \mathrm{~cm}$. A little pale. Gall bladder normal.

Kidney.-Right: Weight, 5 ounces; size, $1.5 \times 5.5 \times 2.5 \mathrm{~cm}$. Left: Weight, $51 / 4$ ounces; size, $12 \times 6.5 \times 2.5 \mathrm{~cm}$. Normal. Capsules strip easily. Markings normal. Both kidneys a little pale. Many mesenteric glands enlarged, the largest about the size of a bean. A number of hemolymph glands enlarged, largest about size of almond. Right adrenal normal.

Stomach.-Very much dilated; contains about 0.5 liter of partly digested food. Mucous membrane of cardia shows some digestion postmortem. Small quantity of food in intestines.
Pure cultures oidium coccidioides obtained from peritoneal fluid and unopened abscesses.

All cultures and specimens were lost in the fire.

\section{CLINICAL SUMMARY OF CASES.}

CASE 1.-Obvious lesions in skin; duration of disease, seven years; patient resided in San Joaquin Valley seven months. Extent of disease: Lymph glands and disseminated nodular lesions in internal organs.

CASE 2.-Obvious lesions in skin, local nine years; duration of disease, 10 years; patient resided in San Joaquin Valley several months. Extent of disease: Lymph glands, many chronic skin abscesses; old scars and consolidated areas in lungs, chronic nodular pleurisy, nodules in diaphragm, circumscribed chronic nodular peritonitis beneath diaphragm and in pelvis, peribronchial and retroperitoneal glands, spleen, adrenals, prostate, both epididymides, testes, seminal vesicles, osteomyelitis in left tibia and metacarpal bone of left hand.

Case 3.-Obvious lesions in skin; duration of disease, three months; patient resided in San Joaquin Valley. Extent of disease: Regionary lymph glands certainly involved and signs of general infection. No autopsy.

Case 4.-Obvious lesions in Iungs; duration of disease, 10 months; patient resided in San Joaquin Valley, but time not known. Extent of disease: Skin, abscess of lung extending up into neck and abscess of liver, lymph glands, osteomyelitis.

Case 5.-Obvious lesions in lungs; patient resided in San Joaquin Valley, but time not known. Extent of disease: Osteomyelitis and periostitis of frontal bone and both tibiæ, suppurative inflammation of right shoulder, both knees, elbows and wrists, abscess from lung extending into mediastinum and diaphragm, liver, retroper it on e a l lymph glands, kid. ney, spleen.

CA S E 6. - No clinical history; no history of residence. Extent of disease: $\mathrm{Lungs}$ peri cardi u m spleen, kidney, meninges.

CASE 7.-Obvious lesions in lungs; duration of dis. ease, three and a half months; patient resided in San Joaquin Valley for twelve days shortly before onset. Extent of disease: Meningitis.

CASE 8.-Obvious lesions in skin; no clinical history; patient resided in San Joaquin Valley. Extent of disease: Lung, spleen, liver, kidney and adrenals.

CASE 9.-Obvious lesions in skin. Duration of disease observed four months; patient discharged improved; resided in San Joaquin Valley several months. Extent of disease: Skin of foot, inguinal and cervical lymph glands, abscess anterior abdominal wall.

CASE 10.--Internal lesions; patient still alive two years later; resided in San Joaquin Valley some months. Extent of disease: Osteomyelitis, foot.

CASE 11.-Internal lesions; probably in lungs; patient supposed to be alive; resided in Sacramento Valley, never in San Joaquin. Extent of disease: Abscess of ribs.

CASE 12.-Internal lesions; probably in lungs. Duration of disease a few months; patient resided in San Joaquin Valley some months; lived in California in course of travels. Extent of disease: Caries at elbow, lungs, probably meninges.

CASE 13.-Wolbach, Jour. Med. Research, 1904, xiii, case not yet published.

CASE 14.-Obvious lesions in lung; Burrow's case (unpublished); duration of disease, eleven months; patient resided in San Joaquin Valley four months. Extent of disease: Multiple abscesses of lung, erosion of sternum, pleurisy with effu. sion, abscess of sacrum, wrist, kidney. Warty nodule on end of nose. 
Case 15.--Obvious lesion in intestinal tract; author's case. Duration of disease two and one-half months; patient resided in San Joaquin Valley five months. Extent of disease: Abscesses with caries of the adjacent bone in the right supraclavicular region, left sternoclavicular articulation, left supraspinous fossa, dorsum of right hand, right ankle, mediastinal glands, peribronchial glands; general involvement of the peritoneum with enlarged mesenteric glands, ulceration on right thigh, left forearm and over right zygoma, on chin.

CASE 16.-J. B., male, aged 28. Family history good; no tuberculosis.

History.-Patient has been two and one-half years in San Joaquin Valley; he worked on irrigating ditches near Fresno. Patient consulted his physician, complaining of general malaise; had no acute symptoms at any time. He has been treated for malaria; large doses of quinin having been admin. istered without effect. He had had a sluggish punched-out ulcer on left wrist; red, with jagged edges; bathed in thin greenish pus; this had partially healed and broken down several times.

Examination.-Under the left clavicle he had an ovoid tumor $4 \times 6 \mathrm{~cm}$., slightly fluctuant and showing no signs of inflammatory process externally; another larger swelling of similar character appeared on the seventh, eighth and ninth ribs in the left parosternal line. On aspiration these tumors contained thin greenish pus loaded with coccidia-like bodies; these had a double contoured capsule but no budding forms; some of these bodies contained spores; with granular protoplasm. Owing to the patient's departure for Oregon, no cultures were made. The patient died in Oregon.

Remarks.-Three years after the first appearance of the ulcer on the wrist, a local physician who attended him in his last illness diagnosed the case as acute miliary tuberculosis. Autopsy showed the Iungs to be studded with what appeared to be miliary tubercles and no further examination was made. No Plasmodia malaria were found. The blood showed an eosinophilia of 12.5 per cent. The tumors had been diagnosed in Fresno as benign fatty tumors.

Since reporting the above cases, I have found two more cases in the service of Drs. E. R. Bryant and H. G. Gates of Los Angeles. The conditions presenting were as follows:

CASE 17.-K. N., Japanese, aged 28. Examined Aly 10, 1906. Employed as a track worker by the Southern Pacific Railway in the San Joaquin Valley for some months preceding his entrance to the hospital. His first symptoms were pain in the neighborhood of the ankle joint, with swelling and redness. Later cervical adenitis. All the lesions suppurated and were treated surgically and healed in from seven to ten days. In a few weeks further abscesses developed in the neighborhood of the former ones and on the face. None of the abscesses was found to connect with the bone. Cultures from a fresh abscess were made and showed the organism characteristic of coccidioidal granuloma. The patient ran a slight fever.

CAsE 18.-T. C., Greek, aged 24. Lived in California three years and in San Joaquin Valley eighteen months. Examined July 10, 1906. Initial symptoms began about June 15, 1906 , with pain in the left axilla simulating pleurisy. Shortly after there appeared a breaking out on the forehead and scalp, pus. tular in character. The skin lesions became covered with thick crust, a cough developed and the patient ran an irregular fever and lost steadily in weight. On examination it was found that the crusts covered small ulcerating surfaces, and when removed a few drops of pus exuded. There were irregular areas of consolidation in the lungs, and loud, moist râles everywhere. Diagnosis was not confirmed by culture examination, but the skin lesions were quite characteristic and the lung condition not to be accounted for by tuberculosis or bronchopneumonia of the usual type.

\section{SUMMARY.}

Specimens of foot amputated by Dr. S. J. Gardner (Case 10) and autopsy specimens of Case 15 with cultures illustrating the morphology of the organisms of oidium coccidioides and blastomycetic dermatitis (obtained through. Morrow from cultures sent him of the Chicago cases) were all destroyed by fire, although I had sets of cultures in three parts of town. The pigs also were destroyed before any definite facts were elicited.

Site of initial lesion:

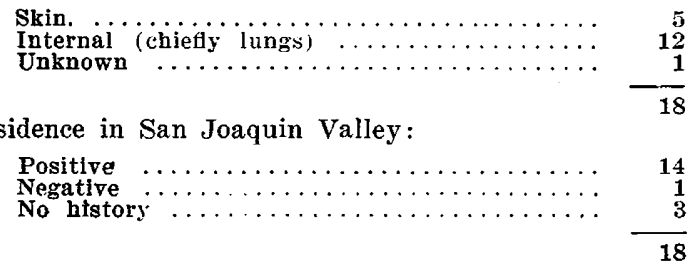

Only one patient is known positively to be alive (Case 10) with local lesions in foot which was promptly amputated. There was no recurrence two years later. Two patients can not be traced. The majority lived less than one year and in all the patients with internal lesions who could be traced the disease was acute from the time the internal lesions began. The disease in California seems to be confined to the lower half of the San Joaquin Valley and has occurred chiefly among men working on railroad construction or irrigating ditches. The patient in the Sacramento case handled raw hides. No case has been found in women. The pericardium and heart alone have escaped involvement in the list of parts affected by the disease. The dissemination in the human being seems to be through both blood and lymph channels.

\section{THE NATURE OF HERPES SIMPLEX, WITH A CONSIDERATION OF ITS DIAGNOSTIC AND PROGNOSTIC SIGNIFICANCE IN VARIOUS INFECTIOUS DISEASES.*}

JAY F. SCHAMBERG, A.B., M.D.

Professor of Dermatology and Eruptive Infectious Diseases in the Philadelphia Polyclinic and College for Graduates in Medicine. PHITADELPHIA.

There are many features of resemblance between simple herpes and herpes zoster, but there are also strong points of dissimilarity. In extreme cases of herpes, particularly about the face, it is sometimes difficult to distinguish between the zoster and simple forms. The clusters of firm vesicles on an erythematous base are identical in both. Histologically, the lesions in the skin and those in the affected nerve structures are practically the same in the two varieties. Thus far simple herpes might almost be regarded as a circumscribed abortive zoster; but there are other differentiating clinical features. The most important is the tendency to recurrence in simple herpes, contrasted with such a rarity of second attacks in herpes zoster as almost to suggest an immunity conferred. Indeed, second attacks of true zoster are no more common than second attacks of measles or scarlet fever. Head and Campbell met with only four cases in 400 cases of zoster. There are certain patients who are so subject to facial herpes that they suffer eight, ten or more attacks a year. In herpes zoster, moreover, there is more pain and the eruption follows in a general way the area of distribution of nerves. In elderly patients neuralgia may persist for weeks, months or years after the disappearance of the eruption.

* Read at the meeting of the Medical Society of the State of Pennsylvania, September, 1906. 